\title{
Pisciculture et environnement : apports de l'analyse du cycle de vie
}

\author{
Joël Aubin \\ Hayo M.G. Van der Werf \\ Inra \\ UMR SAS \\ 65, rue de Saint-Brieuc \\ CS 84215 \\ 35042 Rennes \\ France \\ <aubin@rennes.inra.fr> \\ $<$ Hayo.VanderWerf@rennes.inra.fr>
}

\begin{abstract}
Résumé
L'analyse du cycle de vie (ACV) est une méthode qui permet d'aborder de façon globale les impacts environnementaux associés à la production d'un bien ou d'un service, depuis la production de l'énergie et des intrants jusqu'à sa mise en déchet. L'ACV a été adaptée à la pisciculture et permet de soulever quelques questions clés pour son développement. La comparaison de trois systèmes de production de poissons carnivores en Europe (truite en bassins, turbot en circuit d'eau recirculée et bar en cages) permet de cerner la sensibilité de différents impacts environnementaux de la pisciculture. L'eutrophisation dépend beaucoup de l'indice de conversion et donc de l'efficacité alimentaire du système. L'utilisation d'énergie est fortement associée aux choix de gestion de l'eau et de la production de l'aliment. Le niveau du changement climatique dépend de l'utilisation des ressources énergétiques. Il est très influencé par la production de l'aliment et sa transformation au sein de l'élevage. L'utilisation de production primaire nette (UPPN) estime la quantité de carbone issue de la photosynthèse utilisée pour la production d'un kilogramme de poisson. La comparaison avec les poissons sauvages montre plus de différences entre les systèmes d'élevage et les différentes espèces qu'entre poissons issus de la pêche ou de l'aquaculture. En particulier, la composition des aliments utilisés est discutée. La dépendance à l'eau, c'est-à-dire la quantité d'eau qui traverse l'élevage pour produire un kilogramme de poisson, permet de comparer les élevages et d'apprécier le lien entre l'élevage et le milieu naturel. Les résultats de l'ACV permettent de proposer un profil environnemental relatif des élevages. La pertinence des différents impacts est discutée en regard de la position géographique des élevages associée à la disponibilité en ressources énergétiques et à la sensibilité du milieu.
\end{abstract}

Mots clés : analyse du cycle de vie ; consommation d'énergie ; eutrophisation ; évaluation impact sur environnement ; pisciculture ; productivité primaire.

Thèmes : eau ; pêche et aquaculture ; productions animales ; ressources naturelles et environnement.

\section{Abstract \\ Fish farming and the environment: A life cycle assessment approach}

Life Cycle Analysis (LCA) allows a holistic assessment of the environmental impacts associated with a good or a service, considering its life cycle from the production of its energy and inputs to its disposal. We used LCA to assess the impacts of aquaculture systems and to raise some key questions concerning its development. We compared three production systems of carnivorous fish in Europe: trout in fresh water raceways, turbot in an inland recirculating system and sea bass in sea cages, to explore the environmental impacts of aquaculture. Eutrophication strongly depends on the feed conversion ratio and thus on the efficiency of the system. Energy use is strongly associated with water management options and feed production. Climate change depends on the use of energy resources. It is strongly affected by feed production and feed transformation on the farm. Net primary production use assesses the amount of carbon from photosynthesis used to produce a kilogram of fish. Identifying this impact makes it possible to compare the performance of different farms as well as data on wild fish. Differences between farming systems and among farmed fish species tend to be larger than the differences between fish from aquaculture and fish from fisheries. In particular, the effect of feed composition is discussed. Water dependence, that is to say the amount of water entering the farm per kilo of fish produced, can be used to compare farming systems and assess the links between the farm and its natural environment. LCA results can 
$L^{\prime}$ analyse des liens qui unissent l'aquaculture et l'environnement a longtemps été limitée à l'étude de l'influence des facteurs du milieu sur les performances des élevages aquatiques et, par ailleurs, à l'étude de l'impact du rejet des sites de production sur le milieu naturel environnant. Ces deux thématiques sont toujours étudiées de façon approfondie et séparée. L'aquaculture n'échappe pas aux interrogations qui s'appliquent aux systèmes de production alimentaire et à leur influence à l'échelle globale en lien avec l'accroissement de la population mondiale, la diminution des ressources (énergie fossile, eau douce, écosystèmes marins et terrestres), les dérèglements climatiques et la concurrence entre les activités productives et récréatives. Dans ce contexte, la réponse unifactorielle apportée par la quantification de nutriments et de xénobiotiques émis dans l'eau par les systèmes de production aquacoles n'est plus suffisante pour éclairer les enjeux du développement de cette activité. Il devient nécessaire de prendre en considération la consommation de ressources aussi diverses que l'énergie, l'eau, les protéines, ainsi que l'émission de matières potentiellement polluantes comme les déchets métaboliques, les xénobiotiques, les gaz à effet de serre, etc. Le défi consiste à transformer des informations nombreuses, d'origine diverse et de qualité variable en les synthétisant d'une façon compréhensible, afin de produire un diagnostic et d'orienter des choix techniques ou institutionnels.

Les outils d'analyse environnementale ont cet objectif de synthèse. Parmi ces outils, l'analyse du cycle de vie (ACV) a été retenue comme l'une des méthodes les plus appropriées, car elle permet une approche multicritère, avec des bases scientifiques claires et reconnues pour synthétiser des informations (Van der Werf et Petit, 2002). Cette méthode a déjà été utilisée et adaptée pour les systèmes de production

be used to present a relative environmental profile of farms. The relevance of the different impacts is discussed according to the regional contexts of production which affect availability of energy resources and vulnerability of the environment.

Key words: environmental impact assessment; energy consumption; eutrophication; fish farming; life cycle analysis; primary productivity.

Subjects: animal productions; fishing and aquaculture; natural resources and environment; water.

aquacoles et halieutiques (Seppala et al., 2002 ; Papatryphon et al., $2004 a$; Thrane, 2006 ; Ziegler, 2006).

Dans cet article, nous utilisons le cadre conceptuel de l'ACV pour discuter de certains enjeux de la pisciculture vis-à-vis de sa durabilité environnementale.

\section{Présentation de l'ACV}

L'ACV est une méthode normalisée (ISO 14040-14043, [Jolliet et al., 2005]) conçue pour établir des diagnostics environnementaux de produits ou de services. C'est une méthode d'agrégation des connaissances qui produit des indicateurs d'impacts potentiels à l'échelle locale, régionale ou globale. Elle est basée sur un inventaire de l'ensemble des ressources utilisées (dont l'énergie) et des émissions polluantes, depuis l'extraction des matières premières, pendant l'élaboration du produit, durant son utilisation et lors de sa destruction (mise en déchet, recyclage).

L'ACV définit un cadre méthodologique, dont l'utilisation peut prendre différentes formes. Nous décrivons, ici, la mise en œuvre pratiquée au cours de travaux concernant les systèmes piscicoles.

\section{Définition du champ de l'étude, des limites du système étudié}

L'analyse environnementale de l'activité de production de poisson est généralement réalisée à l'échelle de l'exploitation piscicole. Ce niveau d'analyse doit permettre de montrer la variabilité des impacts environnementaux potentiels en fonction des types de production (espèces, tailles commerciales, etc.), des modes de production (au fil de l'eau, circuits fermés, cages, étangs, etc.) et des choix de gestion technique (niveaux d'intensification, densités, etc.).

Ainsi, le système de production étudié comprend :

- la production et l'acheminement des intrants (aliment, ressources énergétiques, oxygène, traitements vétérinaires), ainsi que leur utilisation sur l'exploitation;

- la production et la transformation des ingrédients composant l'aliment, incluant les phases de pêche et de production agricole ;

- la production des équipements utilisés sur l'exploitation (pompes, trieurs, aérateurs, etc.) ;

- la construction des infrastructures (bâtiments, bassins, etc.) ;

- le transport des éléments entre chaque étape des processus.

\section{Inventaire des extractions et des émissions du système}

Les données concernant les extractions de ressources et les émissions de polluants au niveau de l'exploitation sont relatives à une année ou à un cycle de production et éventuellement basées sur l'élaboration de scénarios alternatifs (objectif de production, densités, introduction de nouvelles techniques, etc.) validés avec l'éleveur.

Les émissions de déchets métaboliques de l'élevage sont estimées grâce à un modèle nutritionnel d'évaluation des rejets (Cho et Kaushik, 1990 ; Bureau et al., 2002 ; Papatryphon et al., 2003), puis confrontées aux valeurs issues des autocontrôles de l'éleveur.

Les informations concernant la production de l'aliment proviennent de données fournies par les fabricants d'aliment et des études publiées (Papatryphon et al., 2004b). Les données sur la production d'oxygène liquide, la fabrication des équipements, les constructions et les transports sont issues d'études antérieures ou d'avis d'experts. 


\section{Évaluation des impacts, par l'agrégation des données}

L'ensemble des données de l'inventaire est ensuite agrégé et exprimé en impacts potentiels à l'aide d'un outil informatique (SIMAPRO). Les différents impacts potentiels sont calculés en agrégeant l'activité de molécules différentes par le biais de facteurs de correspondance, relatifs à une molécule de référence (issus de données scientifiques publiées). Ainsi, les impacts sont exprimés en poids d'équivalent de molécule (exemple : kg éq- $\mathrm{CO}_{2}$ pour le changement climatique).

Les catégories d'impact potentiel jugées comme pertinentes pour l'aquaculture et disponibles actuellement sont les suivantes :

- l'eutrophisation, exprimée en kilogramme d'équivalent phosphates ( $\mathrm{kg}$ éq-PO $\mathrm{PO}_{4}$, concerne les impacts sur les écosystèmes aquatiques et terrestres dus à un enrichissement en azote et phosphore, comme par exemple une prolifération anarchique d'algues qui consomment l'oxygène disponible ;

- le changement climatique, exprimé en kilogramme de dioxyde de carbone ( $\mathrm{kg}$ éq-CO $\mathrm{CO}_{2}$ ), qui évalue la production de gaz à effet de serre par le système ;

- l'utilisation d'énergie, exprimée en mégajoule (MJ), qui regroupe l'ensemble des ressources énergétiques utilisées ;

- l'utilisation de production primaire nette (UPPN), exprimée en kilogramme de carbone ( $\mathrm{kg} \mathrm{C}$ ), qui représente le niveau trophique évalué à partir de la quantité de carbone issue de la production primaire (obtenue par photosynthèse), utilisée par l'élevage.

Toutes ces catégories d'impact sont exprimées par rapport à la production d'une tonne de biomasse de poisson.

\section{Interprétation des résultats}

Afin d'illustrer l'étape d'interprétation, les résultats (basés sur un cycle de production d'un an) de trois fermes de production de poisson carnivores, jugées comme représentatives de trois types de systèmes caractéristiques des tendances de la pisciculture en Europe, seront étudiés :

- une ferme de grossissement de truite arc-en-ciel (Oncorbynchus mykiss) de grande taille $(3 \mathrm{~kg})$ sur rivière en France, produisant 330 tonnes annuellement, avec un aliment commercial (44\% de protéines, $28 \%$ de lipides) et un indice de conversion alimentaire (IC) de 1,21
(IC = quantité d'aliment distribuée divisée par la biomasse de poisson produite) ;

- une ferme de grossissement de turbot de $1,2 \mathrm{~kg}$ (Scophtalmus maximus) en circuit d'eau recirculée en France, produisant 70 tonnes par an, avec un aliment commercial $(55 \%$ de protéines, $12 \%$ de lipides) et un IC de 1,23 ;

- une ferme de production de bars de $350 \mathrm{~g}$ (Dicentrarchus labrax) en cages marines en Grèce, produisant annuellement 256 tonnes, avec un aliment commercial ( $45 \%$ de protéines, $12 \%$ de lipides) et un IC de 1,77.

\section{Réflexions}

\section{pour l'aquaculture}

\section{au travers \\ des indicateurs d'impacts de l'ACV}

\section{Eutrophisation}

L'eutrophisation est directement associée aux rejets de solides et de déchets métaboliques et dépend donc de la qualité et de la valorisation biologique des aliments (Papatryphon et al., 2004b). Ainsi, le premier facteur de variation de ces rejets, dans les systèmes utilisant une alimentation exogène, est l'IC, qui quantifie la performance de l'élevage sur le plan biologique. Il est dépendant des caractéristiques de l'espèce, de l'aliment et de variables techniques, comme la maitrise du rationnement. La comparaison de l'élevage de truite arc-en-ciel en pisciculture en circuit ouvert et celle du bar en cages marines (tableau 1 ; Aubin et al., 2009) montre une différence notable de cet indicateur due à la capacité de transformation de l'espèce (IC du bar plus élevé que celui de la truite) et à la difficulté de contrôler l'appétit et la biomasse de poissons dans des cages. La réalisation effective du phénomène d'eutrophisation demeure, néanmoins, liée à la qualité de l'eau, notamment à la concentration en éléments limitants (azote et phosphore en particulier).

Plusieurs stratégies sont mises en œuvre pour diminuer l'eutrophisation issue des activités de l'élevage :

- l'amélioration des rations, avec des aliments plus digestibles et une alimentation des poissons au plus proche des besoins ;

- le traitement de l'eau issue de la pisciculture en captant les matières solides émises (fèces et aliment non consommé principalement) par des systèmes de décanteur ou de filtration. Malheureusement, ces techniques ne concernent que, de façon marginale, la fraction dissoute des rejets (qui est prédominante pour les rejets azotés), et elles produisent des boues qu'il convient de gérer comme les effluents d'animaux terrestres, généralement par épandage agricole (Petit, 1999) ;

- la valorisation des nutriments par d'autres espèces aquacoles, animales ou végétales.

Il s'agit souvent de polycultures en étangs ; néanmoins des associations d'espèces (poissons, végétaux, mollusques) réparties dans différents compartiments successifs ont aussi été développées (Lefebvre et al., 2001; Troell et al., 2003 ; Neori et al., 2004).

\section{Tableau 1. Eutrophisation (en kilogramme d'équivalent phosphates) pour 1 kilo de poisson, et contribution en pourcentage et en valeur de l'élevage en pisciculture et de la production de l'aliment dans trois systèmes d'élevage.}

Table 1. Eutrophication, for 1 kilo of fish, and contribution in percent and value of onfarm fish production and feed production in three production systems.

\begin{tabular}{lccc}
\hline & $\left.\begin{array}{c}\text { Total } \mathbf{( k g} \\
\text { éq-PO }\end{array} \mathbf{/ k g}\right)$ & $\begin{array}{c}\text { Élevage } \\
\text { du poisson }\end{array}$ & $\begin{array}{c}\text { Production } \\
\text { de I'aliment }\end{array}$ \\
\hline Grande truite d'élevage (France) & 65,9 & $92 \%(60,8)$ & $7 \%(4,7)$ \\
Cages de bars (Grèce) & 108,9 & $93 \%(101,0)$ & $7 \%(7,6)$ \\
Circuit fermé de turbot (France) & 77,0 & $91 \%(69,9)$ & $5 \%(3,9)$ \\
\hline
\end{tabular}




\section{Utilisation d'énergie} pour les productions aquacoles est de plus en plus prise en considération (tableau 2). Les productions de poisson d'aquaculture sont tout à fait comparables à des produits équivalents issus de la pêche, avec une tendance à être moins consommatrices en énergie. C'est, en particulier, le cas pour les poissons marins issus du chalutage, mode de pêche très énergivore (1,5 L de gasoil/kg de poisson (Ziegler, 2006)).

Pour les poissons d'aquaculture, la consommation en énergie a principalement deux origines : l'aliment et la production du poisson sur la ferme (tableau 3). Le poids relatif de la production de l'aliment et de la phase d'élevage sur l'utilisation de l'énergie dépend beaucoup du système technique. Compte tenu de leur similarité, la quantité d'énergie
L'utilisation de ressources énergétiques

nécessaire pour produire les différents aliments est très proche. Par contraste, l'utilisation d'énergie à la ferme est très variable d'un système à l'autre, où les traitements et la mise en mouvement de l'eau (pompage, mise en pression) sont les principaux consommateurs d'énergie. L'oxygène liquide contribue également (environ $10 \%$ ) à l'utilisation d'énergie en pisciculture de truite.

\section{Changement climatique}

Les valeurs de l'impact sur le changement climatique des poissons d'élevage (tableau 4) sont comparables à celles observées pour le cabillaud pêché par chalutage : $4 \mathrm{~kg} \quad \mathrm{CO}_{2}$-eq $/ \mathrm{kg}$ (Ziegler, 2006). Associé principalement à la combustion d'énergie fossile, mais aussi à des émissions de gaz à effet de serre liées aux pratiques agricoles (épandage d'effluent d'élevage, compostage, etc.), le change-

\section{Tableau 2. Utilisation d'énergie (en méga joule, MJ) pour 1 kilo de diverses espèces aquatiques de pêche et d'aquaculture, calculée par analyse du cycle de vie (d'après Mungkung et Gheewala [2007]).}

Table 2. Energy use (in megajoules, MJ) for 1 kilo of various fish products calculated with the LCA method (from Mungkung et Gheewala [2007]).

\begin{tabular}{lcl}
\hline Espèces & $\begin{array}{c}\text { Utilisation } \\
\text { d'énergie } \\
\text { (MJ/kg) }\end{array}$ & \multicolumn{1}{c}{ Référence } \\
\hline Langoustines Suède & 325 & Ziegler (2006) \\
Turbot élevage recirculé & 291 & Aubin et al. (2009) \\
Cabillaud de pêche suédois & 95 & Ziegler (2006) \\
Très grande truite d'élevage en France & 78 & Aubin et al. (2009) \\
Cabillaud de pêche norvégien & 68 & Ellingsen et Aanondsen (2006) \\
Saumon d'élevage norvégien & 66 & Ellingsen et Aanondsen (2006) \\
Bar en cages en Grèce & 55 & Aubin et al. (2009) \\
Crevette d'élevage en Thaïlande & 46 & Mungkung et Gheewala (2007) \\
Truite portion d'élevage en France & 36 & Papatryphon et al. (2004a) \\
\hline
\end{tabular}

\section{Tableau 3. Énergie utilisée (en mégajoules, MJ) pour 1 kilo de poisson, et contribution en pourcentage et en valeur de l'élevage en pisciculture et de la production de l'aliment dans trois systèmes d'élevage.}

Table 3. Energy use (in megajoules, MJ) for 1 kilo of fish, and contribution in percent and value of on-farm fish production and feed production, in three production systems.

\begin{tabular}{lccc}
\hline & $\begin{array}{c}\text { Total } \\
\text { (MJ) }\end{array}$ & $\begin{array}{c}\text { Élevage } \\
\text { du poisson }\end{array}$ & $\begin{array}{c}\text { Production } \\
\text { de I'aliment }\end{array}$ \\
\hline Grande truite d'élevage (France) & 78,2 & $47 \%(36,8)$ & $40 \%(31,3)$ \\
Cages de bar (Grèce) & 54,7 & $17 \%(9,3)$ & $72 \%(39,4)$ \\
Circuit fermé de turbot (France) & 291 & $86 \%(250,3)$ & $11 \%(32,0)$ \\
\hline
\end{tabular}

ment climatique est fortement influencé par la production des aliments. Celle-ci contribue en effet pour 73 et $86 \%$ au changement climatique, respectivement pour la production de grande truite et de bar (tableau 4).

Une part non négligeable des émissions de gaz à effet de serre a lieu à l'échelle de l'élevage. Elle a pour origine principale, l'utilisation de ressources énergétiques au niveau du site. C'est particulièrement le cas pour l'élevage en circuit recirculé, dont $61 \%$ des émissions de gaz à effet de serre sont dues à l'exploitation (tableau 4). Néanmoins, ces résultats sont encore incomplets, car ils ne prennent pas en compte des émissions gazeuses liées au stockage des boues, au fonctionnement des étangs (méthane) et des filtres biologiques pour lesquelles une méthode fiable de quantification n'est pas encore disponible. On peut citer le cas des filtres biologiques où l'existence fortuite de zones anoxiques pourrait induire des productions de $\mathrm{N}_{2} \mathrm{O}$ à fort effet de serre (Aubin et al., 2006). Des travaux complémentaires sont à mener pour mieux caractériser ce type d'émission.

\section{Utilisation de production primaire nette}

Directement liée aux ingrédients des aliments, l'utilisation de production primaire nette (UPPN) est particulièrement sensible à l'efficacité alimentaire du système d'élevage. Le tableau 5 donne quelques repères sur la quantité de production primaire nécessaire à la production de poisson dans le milieu naturel et en conditions d'élevage. Compte tenu de l'imprécision du mode de calcul, ces chiffres ne doivent être pris que comme des repères pour situer les espèces et leurs modes de production (Pauly et Christensen, 1995). Ces chiffres sont assez différents si l'on s'adresse à des espèces de niveau trophique bas (carpe et tilapia), qui sont surtout des consommateurs de végétaux et de plancton ou à des espèces carnivores. Si l'élevage tend à élever l'UPPN, cette tendance n'est pas systématique. En particulier, chez la truite arc-en-ciel d'élevage, on observe des niveaux d'UPPN comparables, voire inférieurs à ceux des salmonidés sauvages. Dans le milieu naturel, les poissons carnivores ont un IC probablement plus élevé qu'en élevage. Par ailleurs, la composition de l'aliment est déterminante. Papatryphon et al. (2004b) ont montré que pour une même teneur en protéines et 
Tableau 4. Changement climatique en kilogramme de dioxyde de carbone pour 1 kilo de poisson, et contribution en pourcentage et en valeur de la consommation énergétique de la pisciculture et de la production de l'aliment dans trois systèmes d'élevage.

Table 4. Climate change, in kilo $\mathrm{CO}_{2}$-eq, for $1 \mathrm{~kg}$ of fish and contribution in percent and value of on-farm energy consumption and feed production, in three production systems.

\begin{tabular}{lcrc}
\hline & $\begin{array}{c}\text { Total } \\
\text { (kg éq-CO }\end{array}$ & $\begin{array}{c}\text { Énergie } \\
\text { pisciculture }\end{array}$ & $\begin{array}{c}\text { Production } \\
\text { de I'aliment }\end{array}$ \\
\hline Grande truite d'élevage (France) & 2,8 & $15 \%(0,4)$ & $73 \%(2,0)$ \\
Cages de bars (Grèce) & 3,6 & $5 \%(0,2)$ & $86 \%(3,1)$ \\
Circuit fermé de turbot (France) & 6,0 & $61 \%(3,7)$ & $32 \%(1,9)$ \\
\hline
\end{tabular}

en lipides, des aliments pour truite, utilisant des intrants d'origines diverses (poisson, productions végétales) pouvaient induire des UPPN de $0,6 \mathrm{~kg}$ C/ $\mathrm{kg}$ (sans ingrédients d'origine poisson), 19,1 kg $\mathrm{C} / \mathrm{kg}$ (niveaux faibles d'ingrédients d'origine poisson) ou $41,3 \mathrm{~kg} \mathrm{C} / \mathrm{kg}$ (forts niveaux d'ingrédients d'origine poisson).

\section{Dépendance à l'eau}

La relation des élevages piscicoles à l'eau est très différente d'un système d'élevage à un autre. Il peut s'agir simplement d'un support physique, d'un véhicule pour les gaz, des produits dissous (ou en suspension) ou d'un milieu productif qui fournit de l'alimentation et assure des fonctions de recyclage. De ce fait, l'eau pose un problème méthodologique dans les ACV des systèmes piscicoles, car elle n'est pas consommée, mais bien souvent ne fait que transiter dans les structures.

En complément des catégories d'impact de l'ACV décrites précédemment, un indicateur "dépendance à l'eau " est proposé. Il mesure l'eau qui traverse le système d'élevage relativement à la biomasse de

Tableau 5. Utilisation de production primaire nette (UPPN) par différentes espèces de poisson en condition sauvage ou d'élevage en kilogramme de carbone par kilogramme de poisson $(\mathrm{kg} \mathrm{C} / \mathrm{kg})$.

Table 5. Net Primary Production Use (NPPU), in kilos of carbon, associated with 1 kilo of different species of fish in natural or farming conditions.

\begin{tabular}{|c|c|c|}
\hline Espèces & $\begin{array}{l}\text { UPPN } \\
(\mathbf{k g ~ C} / \mathbf{k g})\end{array}$ & Référence \\
\hline Truite arc-en-ciel sauvage & 111 & $\begin{array}{l}\text { Halfon et Schito, } 1993 \\
\text { Pauly et Christensen, } 1997\end{array}$ \\
\hline Cabillaud sauvage & 76 & Pauly et Christensen, 1995 \\
\hline Bar en cages en Grèce & 71 & Aubin et al. (2009) \\
\hline Très grande truite d'élevage en France & 62 & Aubin et al. (2009) \\
\hline Turbot élevage recirculé & 61 & Aubin et al. (2009) \\
\hline Saumon atlantique sauvage & 44 & Pauly et Christensen, 1995 \\
\hline Truite portion d'élevage & 44 & Papatryphon et al., 2003 \\
\hline Turbot sauvage & 35 & Pauly et Christensen, 1995 \\
\hline Bar sauvage & 35 & Pauly et Christensen, 1995 \\
\hline Carpes & 5,6 & Pauly et Christensen, 1995 \\
\hline Tilapia et autres cichlidés & 3,5 & Pauly et Christensen, 1995 \\
\hline
\end{tabular}

Valeurs calculées selon la formule de Pauly et Christensen (1995) et les données de niveau trophique de Pauly et Christensen (1995 et 1997). Le niveau élevé d'UPPN, associé à la truite arc-en-ciel, est lié au régime alimentaire particulier de la truite en lac.

poisson produite. De nature très différente, les exemples présentés donnent des résultats très contrastés: $4,8 \mathrm{~m}^{3} / \mathrm{t}$ pour le circuit recirculé de turbot, $52,6 \mathrm{~m}^{3} / \mathrm{t}$ pour la grande truite en bassins, $48780 \mathrm{~m}^{3} / \mathrm{t}$ dans les cages de bar.

Un des écueils de cette comparaison réside dans la nature de l'eau qui est très différente : pour le bar, il s'agit d'eau de mer, ressource considérée comme illimitée, et pour la truite, il s'agit d'eau de rivière avec un débit restreint et qui peut subir de fortes amplitudes au cours de l'année. On peut constater que le circuit recirculé remplit son objectif de limiter sa dépendance au milieu, avec une utilisation d'eau dix fois moins élevée que l'élevage en circuit ouvert.

\section{Comparer les systèmes}

L'ACV permet de comparer des systèmes d'élevage, mais aussi de proposer un profil d'impact environnemental, mettant en évidence les "points critiques". La figure 1 montre le profil environnemental relatif des trois élevages qui nous servent d'exemple. Il en ressort que le système d'élevage de turbot en eau recirculée est surtout caractérisé par un niveau élevé d'utilisation d'énergie et d'impact sur le changement climatique. Le bar en cages est caractérisé par des niveaux élevés d'impact liés à la gestion de son alimentation : UPPN et eutrophisation, ainsi qu'un niveau de dépendance à l'eau très élevé. L'élevage de la truite en bassins semble apparaître comme un compromis parmi ces élevages de poissons carnivores. Néanmoins, pour ce système de production, les questions de l'utilisation de la production primaire, de l'eutrophisation et de la dépendance à l'eau sont à surveiller.

\section{Discussion}

À la lumière de ces résultats, il apparaît qu'il y a plus de variabilité dans les impacts environnementaux entre les différents systèmes ou espèces d'élevage qu'entre les poissons de pêche et les poissons d'aquaculture. Cela semble signifier qu'il y a de la marge pour améliorer le profil environnemental de ces deux moyens de fournir du poisson au consommateur et qu'il est inutile d'opposer ces deux systèmes sur un plan environnemental. La question se pose plutôt en termes de choix, par le consommateur, 


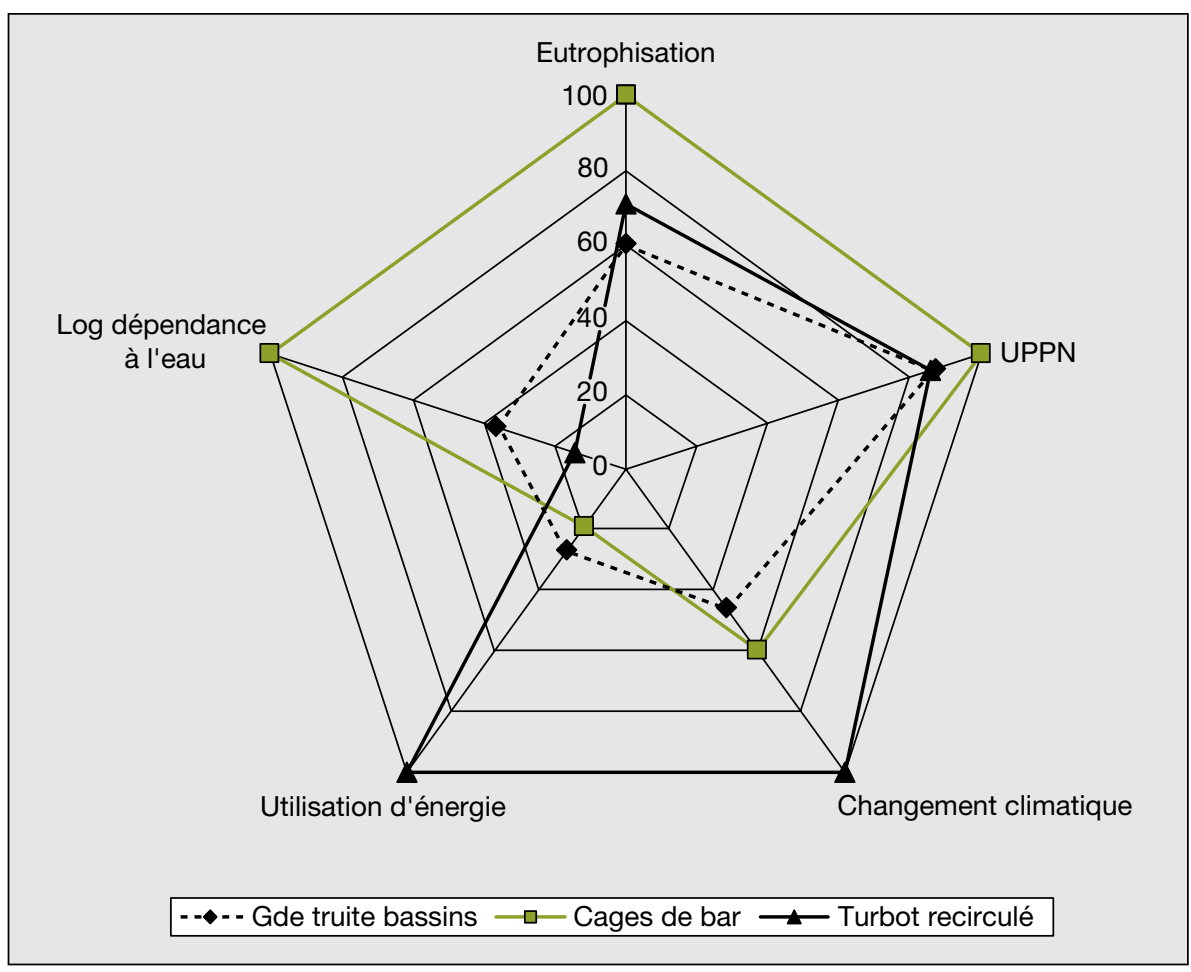

Figure 1. Graphique radial de comparaison relative de six catégories d'impact pour les trois systèmes de production piscicoles.

Figure 1. Radial graph comparing the relative impact, for six impact categories, of three fish production systems.

Les points les plus proches du centre ont l'impact relatif le plus faible. Les valeurs de dépendance à l'eau ont été transformées en log 10.

UPPN : utilisation de production primaire nette.

d'espèces qui sont placées à différents niveaux de la chaîne trophique et se positionnent donc différemment en termes d'efficacité écologique.

Compte tenu des différences modérées en termes d'UPPN parmi les poissons carnivores de pêche ou d'aquaculture, la question de l'élevage de ces espèces se pose plus en termes de positionnement géographique. Actuellement, les zones de production de poisson d'élevage sont éloignées des zones d'origine des intrants (huiles et farines de poisson et produits agricoles), et les rejets en nutriment des élevages ne contribuent que très partiellement à la consolidation de ces mêmes ressources (les éléments nutritifs émis par les élevages ne sont pas recyclés dans le système de production des intrants). Contrairement aux systèmes d'élevage en étangs, où la fonction de recyclage est traditionnellement mise en avant, les systèmes d'élevage de poissons carnivores émettent directement dans le milieu naturel des quantités d'éléments nutritifs qui représentent une perte économique importante, à double titre :
- ils ne sont pas valorisés sur le plan économique ;

- ils nécessitent de mettre en œuvre des installations coûteuses en énergie pour leurs traitements (qui demeurent partiels). Par ailleurs, ces apports de matières solides ou dissoutes modifient de façon plus ou moins transitoire le milieu aquatique récepteur, qui est chargé de les transformer et de les épurer. La sensibilité du milieu récepteur est pour ces systèmes un facteur clé, car elle va conditionner l'apparition d'impacts visibles sur les écosystèmes. La localisation des élevages piscicoles dans des zones plus ou moins sensibles aux phénomènes d'eutrophisation est donc très importante. À titre d'exemple, l'élevage de bar, situé en mer Méditerranée, qui est oligotrophe, présente un risque d'eutrophisation très faible mais aura plutôt un effet sur le benthos (Karakassis et al., 2000). À l'opposé, un élevage de truite en région atlantique présente un risque important de participer à l'eutrophisation (marées vertes) en zone littorale où les sédiments marins sont très chargés en phosphore, issu des activités humaines
(Ménesguen et al., 2001). Néanmoins, l'élevage de bar ne sera pas sans impact sur l'écosystème méditerranéen. Il peut augmenter la productivité des populations de poissons naturellement présentes dans le milieu (Machias et al., 2004), mais aussi diminuer la croissance et la survie des champs de posidonies (Posidonia oceania) jusqu'à $800 \mathrm{~m}$ des cages (Marbà et al., 2006), et ainsi menacer l'existence de ces milieux oligotrophes très particuliers.

Un autre effet de la localisation géographique des élevages piscicoles réside dans la disponibilité et la nature de l'énergie utilisée sur le site. Les impacts associés à la production et à l'utilisation des différentes ressources énergétiques (charbon, pétrole, uranium, hydroélectricité) sont très différents (principalement changement climatique). En particulier, il faut remarquer la grande différence entre les systèmes de production piscicoles utilisant l'électricité en France (à $86 \%$ d'origine nucléaire) et dans certains pays méditerranéens où elle est principalement liée à la combustion de produits pétroliers (la Grèce, par exemple). La localisation est aussi un point sensible pour les systèmes basés en mer (ou de la pêche), où la distance des sites à la côte influe directement sur l'énergie utilisée par les élevages, majoritairement due aux transports par bateau du matériel, de l'aliment et des poissons.

\section{Conclusion}

Basée sur des indicateurs d'impacts environnementaux qui demeurent potentiels, l'ACV permet d'identifier les différentes caractéristiques des systèmes de production qui influent sur leur efficacité. Elle oblige à représenter l'élevage de façon plus systémique en élargissant la réflexion à la fourniture des intrants et à la production de ceux-ci. La contribution des différentes parties du système aux impacts peut être estimée, et la méthode permet d'évaluer la pertinence environnementale des alternatives techniques. Les échelles d'espace et la localisation géographique sont importantes afin de discuter de la pertinence des catégories d'impact et pour mieux prendre en compte les contextes économiques et environnementaux des élevages.

L'ACV est donc un bon support de réflexion et de discussion afin de reposi- 
tionner les enjeux des systèmes d'élevages piscicoles entre eux, en comparaison avec d'autres moyens de fournir un produit similaire (comme le poisson de pêche) ou d'autres produits alimentaires. Néanmoins, l'ACV doit encore progresser afin de mieux prendre en compte d'autres catégories d'impact qui sont au cour des questions d'environnement, actuellement comme l'écotoxicité ou la biodiversité. Par ailleurs, il est nécessaire de multiplier les études de cas afin de mieux cerner la variabilité interne associée à un même système de production.

\section{Références}

Aubin J, Papatryphon E, Van der Werf HMG, Petit J, Morvan YM. Characterisation of the environmental impact of a turbot (Scophthalmus maximus) re-circulating production system using Life Cycle Assessment. Aquaculture $2006 ; 261$ : 1259-68.

Aubin J, Papatryphon E, Van der Werf HMG, et al. Assessment of the environmental impact of carnivorous finfish production systems using Life Cycle Assessment. J Cleaner Pro duction 2009 ; 17 : 354-61.

Bureau DP, Gunther S, Cho CY. Chemical composition and preliminary theoretical estimates of waste outputs of rainbow trout reared in commercial cage culture operations in Ontario. North Am J Aquac 2002 ; 65 : 33-8.

Cho CY, Kaushik SJ. Nutritional energetics in fish: energy and protein utilization in rainbow trout (Salmo gairdneri). World Rev Nutr Diet $1990 ; 61: 132-72$.

Ellingsen $\mathrm{H}$, Aanondsen SA. Environmental impacts of wild caught cod and farmed salmon - a comparison with chicken. Int J Life Cycle Assess $2006: 11: 60-5$.
Halfon $E$, Schito N Lake Ontario food web, an energetic mass balance. In: Christensen V, Pauly D, eds. Trophic models of aquatic ecosystems. ICLARM Conf. Proc. 26. 1993; In : Pauly, D, Christensen V. Trophic level of fishes. In : Froese R, Pauly D, eds. Fishbase 97: concepts, design and data sources. Manilla: ICLARM, Manilla, 1997.

Jolliet O, Saadé M, Crettaz P. Analyse du cycle de vie. Comprendre et réaliser un écobilan. Lausanne (Suisse): Presses polytechniques et universitaires romandes, 2005.

Karakassis I, Tsapakis M, Hatziyanni E, Papadopuolou KN, Plaiti W. Impact of cage farming of fish on the seabed in three Mediterranean coastal areas. ICES JMar Sci 2000; 57 : 1462-71.

Lefebvre S, Bacher C, Meuret A, Hussenot J. Modelling nitrogen cycling in a mariculture ecosystem as a tool to evaluate its outflow. Estuar Coast Shelf Sci 2001 ; 52 : 305-25.

Machias A, Karakassis I, Labropoulou M, Somarakis S, Papadopoulou KN, Papaconstantinou C. Changes in wild fish assemblages after the establishment of a farming zone in an oligotrophic marine ecosystem. Estuar Coast Shelf Sci 2004 ; 60 : 771-9.

Marbà N, Santiago R, Diaz-Almela E, Alvarez EM, Duarte C. Seagrass (Posidonia oceanica) vertical growth as an early indicator of fish farm-derived stress. Estuar Coast Shelf Sci $2006 ; 67: 475-83$

Ménesguen A, Aminot A, Belin C, et al. L'eutrophisation des eaux marines et saumâtres en Europe, en particulier en France. Rapport IFREMER DEL/EC/01.02 pour la Commission Européenne - DG.ENV.B1. Brest : Ifremer, 2001.

Mungkung R, Gheewala SH. Use of Life Cycle Assessment (LCA) to compare the environmental impacts of aquaculture and agri-food products. In: Comparative Environmental Costs of Aquaculture and Other Food Production Sectors. FAO Technical Report. Rome: FAO, 2007

Neori A, Chopin T, Troell M, et al. Integrated aquaculture: rationale, evolution and state of the art emphasizing seaweed biofiltration in modern mariculture. Aquaculture 2004; 231 : 361-91.
Papatryphon E, Petit J, Van der Werf HMG. The development of Life Cycle Assessment for the evaluation of rainbow trout farming in France. Proceedings of the 4th International Conference on Life Cycle Assessment in the Agrifeed sector. 6-8 October 2003, Horsens, Denmark, 2004a.

Papatryphon E, Petit J, Kaushik SJ, Van der Werf HMG. Environmental impact assessment of salmonids feeds using Life Cycle Assessment. Ambio $2004 ; 33: 316-23$

Papatryphon E, Petit J, Van der Werf HMG, Kaushik SJ, Kanyarushoki C. Nutrient balance modelling as a tool for environmental management in aquaculture: the case of trout farming in France. Environ Manage 2003; 35 : 161-74.

Pauly D, Christensen V. Primary production required to sustain global fisheries. Nature 1995 ; $374: 255-7$.

Pauly D, Christensen V. Trophic level of fishes. In: Froese R, Pauly D, eds. Fishbase 97: concepts, design and data sources. Manilla: ICLARM, 1997.

Petit J. Environnement et aquaculture, tome 1 aspects techniques et économiques. France: Inra éditions, 1999

Seppala J, Silvenius F, Gronroos J, Makinen T, Silvo K, Storhammar E. Rainbow trout produc tion and the environment. Helsinki: Finnish Environmental Institute, 2002.

Thrane M. LCA of Dansih Fish Products, New methods and insights. Int J LCA 2006; 11 : 66-74.

Troell M, Halling C, Neori A, et al. Integrated mariculture: asking the right questions. Aquaculture $2003 ; 226$ : 69-90.

Van der Werf HMG, Petit J. Evaluation of the environmental impact of agriculture at the farm level: a comparison and analysis of 12 indicator-based methods. Agric Ecosyst Environ $2002 ; 93: 131-45$.

Ziegler F. Environmental Life Cycle Assess ment of seafood products from captures fisheries. Göteborg: Department of Marine Ecology, Götborg University, SIK, 2006. 